\title{
Ventromedial Preoptic Prostaglandin E2 Activates Fever-Producing Autonomic Pathways
}

\author{
Thomas E. Scammell, Joel K. Elmquist, John D. Griffin, and Clifford B. Saper \\ Department of Neurology and Program in Neuroscience, Beth Israel Hospital and Harvard Medical School, Boston, \\ Massachusetts 02215
}

Fever is thought to be initiated by pyrogenic cytokines inducing the production of prostaglandin E2 (PGE2) in the preoptic area (POA); PGE2 may act as a paracrine mediator that stimulates the neural pathways that raise body temperature. This essential role for prostaglandins in fever first was proposed 25 years ago, but the specific preoptic cell groups at which PGE2 acts and the pathways through which fever is produced remain poorly understood. To better define the role of preoptic PGE2 in fever, we developed a new method for combining acute brain injections with Fos immunohistochemistry. We microinjected a threshold dose of PGE2 to construct an anatomically detailed map of fever-producing preoptic sites. The most pyrogenic preoptic sites were clustered along the ventromedial aspect of the POA, surrounding and just anterior to the organum vasculosum of the lamina terminalis. We then used Fos immunohis- tochemistry to identify the pattern of neural activation induced by fever-producing preoptic injections of PGE2 and compared it with the Fos pattern seen after systemic immune stimulation. PGE2 fever was accompanied by Fos induction in the ventromedial POA and the parvicellular subnuclei of the paraventricular nucleus of the hypothalamus (PVH). In contrast to the Fos pattern seen after intravenous lipopolysaccharide administration, PGE2 injection did not induce Fos in the circumventricular organs or the magnocellular subnuclei of the PVH. These observations establish a potential site of PGE2 action during fever and help define candidate pathways through which fever occurs.

Key words: fever; prostaglandin E2; body temperature; thermoregulation; autonomic system; Fos; preoptic area; hypothalamus
Fever is produced by the coordinated actions of many CNS regions as an adaptive response to infection. Traditionally, fever is thought to be initiated by pyrogenic cytokines acting on the preoptic area (POA). Specifically, these cytokines are thought to induce the production of prostaglandin E2 (PGE2) in the POA, which then stimulates the neural pathways that raise body temperature $(\mathrm{Tb})$ (for review, see Kluger, 1991; Saper and Breder, 1994).

This essential role for prostaglandins in fever first was proposed 25 years ago to explain the observation that systemic inhibition of prostaglandin production blocked fever (Vane, 1971). Although much research now suggests that preoptic PGE2 is required for fever, previous studies have been unable to determine the specific sites at which PGE2 acts or to verify that PGE2 actually produces fever through the same pathways as fever induced by immune stimulation. Most previous studies of PGE2 fever have involved injection of relatively large doses of PGE2 (typically >100 ng), and it is possible that the fevers observed occurred through mechanisms not typically engaged during natural fever. Nearly all of these studies used chronic injection cannulae, which, like any foreign body, produce local inflammation and PGE2 production (Yergey and Heyes, 1990). Additionally, these experiments lacked adequate anatomic resolution, because they used relatively large volumes of PGE2 ( $\geq 1 \mu \mathrm{l})$, which simultaneously could stimulate a large volume of preoptic tissue. Although many of these studies helped focus attention on the POA (Feldberg and Saxena, 1971; Williams et al., 1977; Stitt, 1991), the specific preoptic cell groups

Received May 30, 1996; revised July 10, 1996; accepted July 15, 1996.

Correspondence should be addressed to Dr. Thomas Scammell, Department of Neurology, Beth Israel Hospital, 77 Avenue Louis Pasteur, Boston, MA 02115.

Copyright (C) 1996 Society for Neuroscience $0270-6474 / 96 / 166246-09 \$ 05.00 / 0$ at which PGE2 acts and the pathways through which fever is produced remain unknown.

To better define the role of preoptic PGE2 in fever, we have combined an acute, minimally traumatic brain injection technique with Fos immunohistochemistry. The immediate early gene product Fos is a sensitive marker of neuronal activation often used functionally to identify extended neural systems activated by specific stimuli (Sagar et al., 1988; Morgan and Curran, 1991). Initially, we determined the minimum dose of PGE2 required for induction of fever, and we used this threshold dose to construct an anatomically detailed map of fever-producing preoptic sites. We then used Fos immunohistochemistry to identify the candidate circuits activated by fever-producing preoptic injections of PGE2 to determine how well they corresponded with the Fos pattern seen after immune stimulation (Elmquist et al., 1996).

\section{MATERIALS AND METHODS}

Animals. Pathogen-free, male Sprague Dawley rats (Taconic, Germantown, NY) weighing 280-350 gm were used in this study. Rats were housed individually in a pathogen-free barrier facility with unrestricted access to food and water in a room maintained at $21.5-22.5^{\circ} \mathrm{C}$. Lights turned on at 7 A.M. and off at 7 P.M. All protocols were approved by the Institutional Animal Care and Use Committees of Beth Israel Hospital and Harvard Medical School. Each rat was used only once. Sixty-two rats were used in these experiments, and 15 others were excluded from the study because of occluded or missing catheters (14 rats) or temperature transmitter failure (1 rat).

Procedures and analysis. In experiment 1, we acutely injected 1-100 ng of PGE2 into the POA of briefly anesthetized rats to determine the minimum fever-producing dose of PGE2 in our model.

In experiment 2, we injected the threshold pyrogenic dose of PGE2 (1 ng) into the POA to determine the anatomic distribution of PGE2sensitive sites. $\mathrm{Tb}$ was recorded for $2 \mathrm{hr}$, and febrile responses to PGE2 injections in different preoptic regions were compared. 
In experiment 3, we studied the pattern of Fos expression in brain induced by fever-producing POA injections of PGE2. Vehicle or a high (100 ng) or low (1 ng) dose of PGE2 was acutely injected into the ventromedial region of the POA. Tb was recorded for $2 \mathrm{hr}$, and the brains then were processed for Fos immunohistochemistry. To determine quantitatively the effects of PGE2 injection, Fos-immunoreactive nuclei were counted within three key autonomic sites that are activated during the fever produced by systemic immune stimulation: the ventromedial preoptic (VMPO) area, the paraventricular nucleus of the hypothalamus $(\mathrm{PVH})$, and the nucleus of the solitary tract (NTS) (Sagar, 1994; Elmquist et al., 1996).

Placement of intravenous catheters and telemetry devices. Telemetry was used for monitoring $\mathrm{Tb}$, and chronic intravenous catheters were used for injection of the general anesthetic just before brain injection. All temperature transmitters (type VM-FH, Mini Mitter, Sun River, OR) were calibrated in a warm water bath at temperatures between 35 and $39^{\circ} \mathrm{C}$ as detailed in the manufacturer's instructions. Five to seven days before the experiment, rats were anesthetized with chloral hydrate $(350 \mathrm{mg} / \mathrm{kg}$, i.p.), and SILASTIC catheters were inserted into the femoral vein up to the level of the right atrium. The free end of the catheter was externalized at the interscapular area, flushed with $0.1 \mathrm{ml}$ of heparinized $(10 \mathrm{U} / \mathrm{ml})$ pyrogen-free $0.9 \%$ saline (Sigma, St. Louis, MO), and plugged with a sterile wire stylet. Catheters were flushed again $2 \mathrm{~d}$ before the experiment to ensure patency. After insertion of the catheter, a temperature transmitter was placed into the peritoneal cavity via a midline incision. Tb signals were received by an antenna below the rat's cage and relayed to a signal processor connected to a Compaq 486 PC. Monitoring of Tb began at least $12 \mathrm{hr}$ before drug injection to assess baseline Tb. Average baseline $\mathrm{Tb}$ was $37.1^{\circ} \mathrm{C}$, and baseline $\mathrm{Tb}$ did not differ significantly among groups. $\mathrm{Tb}$ data are presented as the change in temperature from the average baseline over the hour preceding the brain injection.

$P O A$ injections. On the day of the experiment, each rat was anesthetized with the short-acting general anesthetic propofol $(10 \mathrm{mg} / \mathrm{kg}$, i.v. $)$ (Zeneca, Wilmington, DE) (Larsson and Wahlstrom, 1994) between 9 A.M. and 10 A.M. The rat was placed in a stereotaxic frame on a $36^{\circ} \mathrm{C}$ heating pad, and 10-100 $\mathrm{nl}$ of PGE2 solution or vehicle was microinjected into the POA using a previously described air pressure injection apparatus (Amaral and Price, 1983). The silane-coated glass pipette was introduced at a $7^{\circ}$ angle off vertical to avoid the sagittal sinus, and injection coordinates varied from $0.25 \mathrm{~mm}$ anterior to bregma to $0.6 \mathrm{~mm}$ posterior to bregma, from 1 to $3 \mathrm{~mm}$ lateral to the midline, and from 6.2 to $8.8 \mathrm{~mm}$ below the dural surface, according to the atlas of Paxinos (Paxinos and Watson, 1986). The vehicle for these POA injections was pyrogen-free $0.9 \%$ saline containing $1 \% 0.1 \mu \mathrm{m}$ rhodamine-labeled latex microspheres (Molecular Probes, Eugene, OR) for injection-site identification. PGE2 (1 mg) (Sigma) was dissolved in vehicle to a a final concentration of $100 \mathrm{ng} / 100 \mathrm{nl}$ for experiments 1 and 3 (100 $\mathrm{ng}$ dose) and $1 \mathrm{ng} / 10 \mathrm{nl}$ for experiments 2 and 3 ( $1 \mathrm{ng}$ dose). Anesthesia was adjusted individually, and each rat often required a small supplemental dose of propofol (typically $2.5-5.0 \mathrm{mg} / \mathrm{kg}$ ) 7-10 min after induction. Therefore, the total dose of propofol varied among animals, but there was no correlation between total propofol dose and the subsequent $\mathrm{Tb}$ response. After the brain injection, the intravenous catheter was flushed, the scalp wound was closed with surgical clips, and the rat was returned to its cage. The entire procedure was completed in $10-15 \mathrm{~min}$, and rats recovered rapidly, usually resuming normal activity and grooming within 5-10 min after returning to their cages. Tb was recorded over the next $2 \mathrm{hr}$.

Perfusion and histology. After the $2 \mathrm{hr}$ monitoring period, the animals were deeply anesthetized with chloral hydrate $(500 \mathrm{mg} / \mathrm{kg}$, i.p.) or propofol $(20 \mathrm{mg} / \mathrm{kg}$, i.v. $)$ and perfused transcardially with $0.9 \%$ saline for $5 \mathrm{~min}$, followed by $500 \mathrm{ml}$ of phosphate-buffered $4 \%$ paraformaldehyde, $\mathrm{pH} 7.0$. The brains were removed, stored in the same fixative for $4 \mathrm{hr}$, and submerged in $20 \%$ sucrose, and four series of coronal sections were cut at $40 \mu \mathrm{m}$ on a freezing microtome. Serial sections through the POA were examined under fluorescence microscopy. The center of the injection site was defined as the largest cluster of fluorescent spheres and drawn with a camera lucida. The sections were stored at $4^{\circ} \mathrm{C}$ in tissue culture dishes containing $0.02 \%$ sodium azide in PBS until immunohistochemical staining was initiated.

Fos immunohistochemistry was performed as described previously (Elmquist et al., 1996). Briefly, sections were incubated for $48 \mathrm{hr}$ at room temperature with a rabbit primary antiserum (Oncogene, Cambridge, MA) $[1: 10,000$ dilution in PBS with 3\% normal goat serum and $0.25 \%$ Triton X-100 (PGT)] for the N-terminal domain of Fos with no known cross-reactivity with any identified Fos-related antigens. Sections were subsequently incubated for $2 \mathrm{hr}$ with a biotinylated goat anti-rabbit IgG (Vector, Burlingame, CA) (1:600 dilution in PGT) and then reacted with avidin-biotin complex (Vector Elite Kit, 1:200 dilution in PBS). A combination of $0.04 \%$ diaminobenzidine tetrahydrochloride (Sigma), $0.01 \%$ hydrogen peroxide, and $0.01 \%$ cobalt chloride was used for the chromogen reaction. Controls for the specificity of the antiserum consisted of incubation of the tissue in antiserum that had been preadsorbed with the Fos antigen (peptide-2, Oncogene) $(15 \mu \mathrm{M})$. In addition, negative controls were generated by omission of the primary antiserum.

Data analysis. Sections were observed with a Leitz Laborlux microscope for neurons containing Fos-like immunoreactivity (Fos-IR), and the pattern of labeling was mapped. In addition, we quantified the relative number of cells containing Fos-IR for rats that received $100 \mathrm{ng}$ of PGE2 $(n=5)$ or $100 \mathrm{nl}$ of vehicle $(n=3)$. Three key thermoregulatory regions were analyzed: VMPO, PVH, and NTS. In the $1 \mathrm{ng}$ PGE2 experiment, cells were counted only in the VMPO, because little change in Fos-IR was evident in other regions. Animals in this series received $1 \mathrm{ng}$ of PGE2 into pyrogenic $(n=4)$ or nonpyrogenic $(n=3)$ sites or vehicle into pyrogenic $(n=3)$ or nonpyrogenic $(n=4)$ sites. A treatment-blinded examiner counted clearly stained Fos-immunoreactive nuclei in one section per animal for each of the three regions. In the VMPO and PVH, nuclei were counted opposite the side of injection to minimize counting any Fos-IR that may have been caused by pipette trauma. Using a $10 \times$ objective, cells were counted in the VMPO $160 \mu \mathrm{m}$ caudal to the organum vasculosum of the lamina terminalis (OVLT) coincident with the opening of the third ventricle (see Fig. 5). Cells were counted in the PVH at the level of the center of the posterior magnocellular subnucleus and in the NTS at the most rostral level of the area postrema. The full extent of the PVH and the NTS was analyzed, but because the boundaries of the VMPO are less distinct, a $500 \times 400 \mu \mathrm{m}$ grid was used to delineate the area of quantification. The grid was located such that the medial edge abutted the third ventricle and extended $500 \mu \mathrm{m}$ laterally and $200 \mu \mathrm{m}$ above and below the ventrolateral corner of the third ventricle (Elmquist et al., 1996).

Statistical analysis. Tb data are presented as the mean \pm SEM change in temperature in each 10 min interval relative to baseline (average temperature over the hour preceding injection). To determine quantitatively whether febrile responses differed among preoptic regions in experiment 2 (the $1 \mathrm{ng}$ PGE2-mapping experiment), injection sites were categorized into one of seven possible regions (see Fig. 6): (1) the peri-OVLT region (three areas, OVLT, VMPO, and anteroventral periventricular nucleus, within $500 \mu \mathrm{m}$ of the core of the OVLT) $(n=6)$; (2) the meningeal strand, which supports the optic chiasm just rostral to the OVLT or the cell-sparse parenchyma immediately above the strand $(n=6)$; (3) the median preoptic (mnPO) nucleus $(n=6)$; (4) the vertical limb and the horizontal nucleus of the diagonal band $(n=5)$; $(5)$ the medial or lateral POA $(n=7)$; (6) the subarachnoid space or third ventricle $(n=5)$; and (7) other sites $>1 \mathrm{~mm}$ from the OVLT not listed above $(n=13)$ (these included the periventricular preoptic nucleus, perifornical region, medial septum, and other sites). The mnPO is traditionally considered part of the peri-OVLT region, but we have separated it in our analysis to determine whether it might differ functionally. Preliminary experiments had indicated that the largest fevers were evident $30 \mathrm{~min}$ after the PGE2 injection, and, therefore, we chose to compare groups at this time point. To determine the effects of PGE2 treatment, each group was compared with the vehicle-injected group $(n=$ 13). To establish anatomic specificity, each group of injection sites also was compared with the group of 13 injection sites $>1 \mathrm{~mm}$ from the OVLT. Groups were compared using a one-way ANOVA (SYSTAT, SPSS, Chicago, IL) with a Bonferroni correction; $p$ was considered significant if $<0.025$, because two ANOVAs were performed.

In experiment 3 , counts of Fos-immunoreactive nuclei in the $100 \mathrm{ng}$ PGE2 group were compared with the $100 \mathrm{nl}$ vehicle-injected group using $t$ tests with a Bonferroni correction; $p$ was considered significant if $<0.017$, because three regions were studied. In the 1 ng PGE2 experiment, Fos-IR in the VMPO was analyzed using a two-way ANOVA to determine the relative contributions of drug treatment (PGE2 vs vehicle) and injection location (inside or outside the ventromedial pyrogenic zone). Counts of Fos-immunoreactive nuclei were not corrected for double-counting errors (Konigsmark, 1970), because there was no change in sizes of labeled structures among groups and only relative, not absolute, values were sought. 


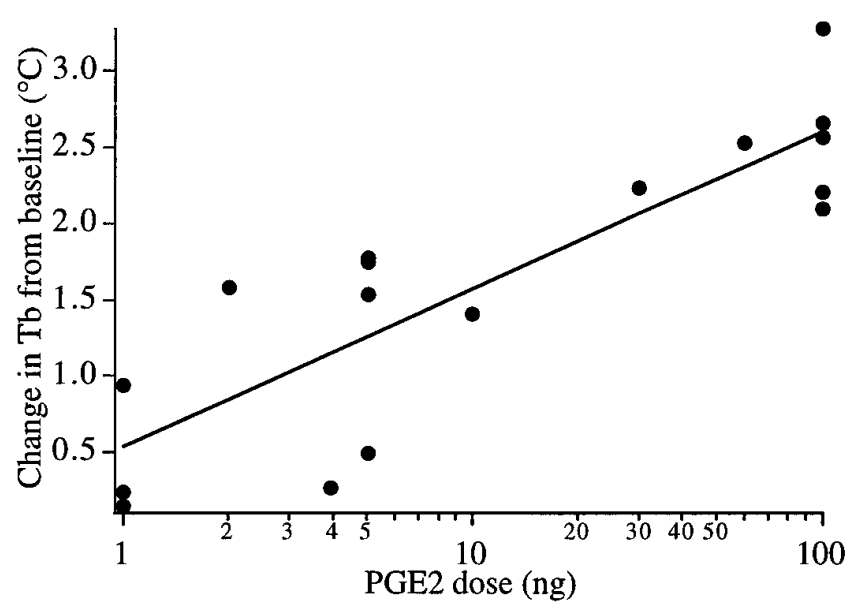

Figure 1. PGE2 injected into the POA induces dose-dependent fever. Each point represents the change in $\mathrm{Tb}$ from baseline of one rat $30 \mathrm{~min}$ after injection. The line demonstrates the best-fit linear regression $(r=0.85)$.

\section{RESULTS}

\section{PGE2 dose-response relationship}

We first determined the minimum amount of PGE2 required to produce fever in our model. Injections of 1-100 $\mathrm{nl}$ of a $1 \mathrm{ng} / \mathrm{nl}$ PGE2 solution targeted at the peri-OVLT region produced doseand volume-dependent fevers (Fig. 1). Injections of $100 \mathrm{ng}$ of PGE2 produced very large fevers 30 min after injection, whereas 1 ng of PGE2 produced small, statistically insignificant increases in $\mathrm{Tb}$ except for one injection near the meningeal strand supporting the optic chiasm. Therefore, $1 \mathrm{ng}$ of PGE2 was determined to be the threshold dose for producing fever, and a more dilute solution of PGE2 (1 ng/10 nl) was used in subsequent experiments to improve the reproducibility of these small injections.

\section{Preoptic sites responsive to PGE2}

We then mapped the sites at which 1 ng of PGE2 produced fever. Because we used fine $(\sim 30 \mu \mathrm{m}$ tip) glass micropipettes, tissue injury was rarely visible within the diencephalon, and injection sites were identified by a tiny cluster of fluorescent latex microspheres (Fig. 2). PGE2 fever was maximal about $30 \mathrm{~min}$ after injection, and this time point was chosen for analysis. Injection of vehicle produced a small, insignificant drop in $\mathrm{Tb}\left(0.15 \pm 0.31^{\circ} \mathrm{C}\right.$ below baseline), and, therefore, fever was defined as a $\mathrm{Tb}$ increase of at least $0.5^{\circ} \mathrm{C}$ above baseline ( $>2$ SDs above the vehicle response). PGE2 was administered to 48 rats, and 19 developed fever. The 10 largest fevers occurred after PGE2 injection into three areas: the peri-OVLT region, the mnPO nucleus, or the meningeal strand that supports the optic chiasm and the parenchyma immediately above the strand (Fig. 3). The febrile response among these three regions was indistinguishable but clearly greater than the $\mathrm{Tb}$ response to vehicle $(p<0.001$ for each of the three groups). These three regions also differed significantly from the group of PGE2 injections $>1 \mathrm{~mm}$ away from the OVLT $(p<$ $0.001)$. The only other injections that produced a slight increase in $\mathrm{Tb}$ were those into the third ventricle or subarachnoid space, but these were not statistically different from vehicle injections.

Injections were categorized as producing no fever $\left(<0.5^{\circ} \mathrm{C}\right)$, small fever $\left(0.5-1.0^{\circ} \mathrm{C}\right)$, or large fever $\left(>1.0^{\circ} \mathrm{C}\right)$ and mapped onto a series of POA drawings (Fig. 4). Injections into the diagonal band nucleus or the subarachnoid space $>500 \mu \mathrm{m}$ rostral to the OVLT had no significant effect on Tb. However, injections 200-

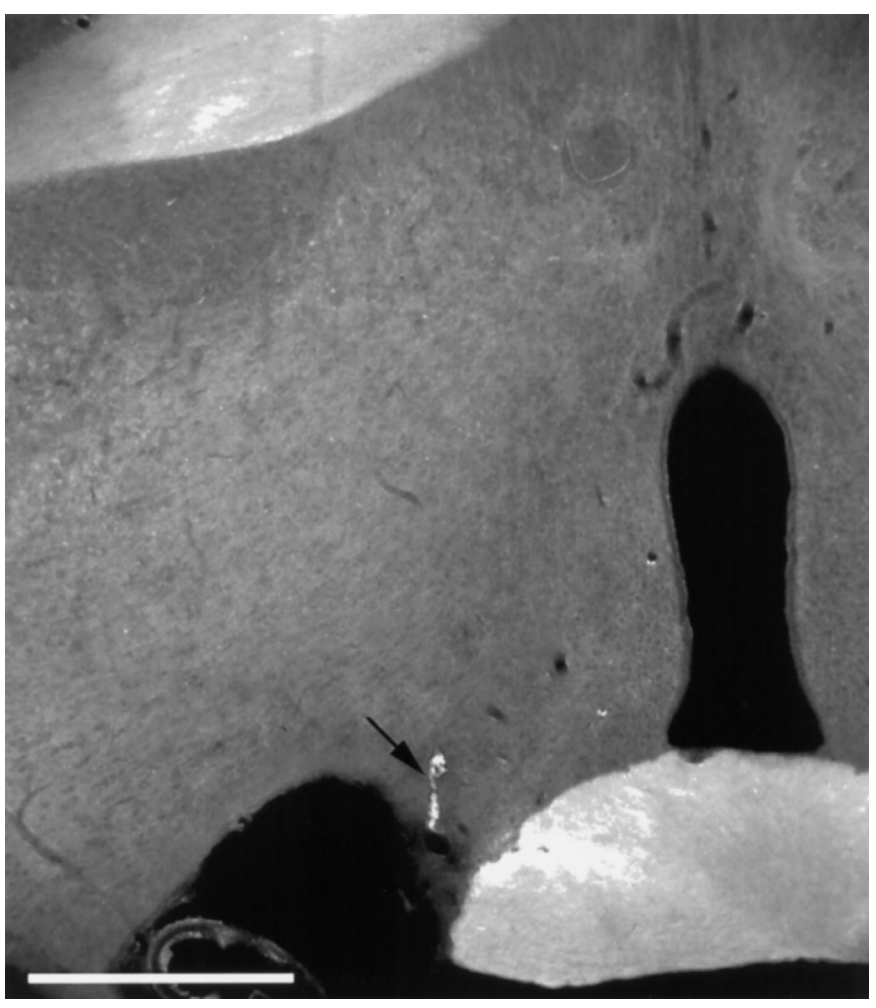

Figure 2. A composite photomicrograph of a representative PGE2 injection (100 ng of PGE2 in $100 \mathrm{nl}$ of vehicle). The injection site (black arrow) is evident as a cluster of fluorescent beads on the boundary between the VMPO and the ventrolateral POA. An image of the rhodamine-labeled microsphere epifluorescence was digitally superimposed on the corresponding dark-field image. Scale bar, $500 \mu \mathrm{m}$.

$300 \mu \mathrm{m}$ caudal, into the cell-sparse zone below the diagonal band or into the meningeal strand supporting the optic chiasm just anterior to the OVLT, produced large fevers. Injections into the VMPO, anteroventral periventricular nucleus, or the mnPO nu-

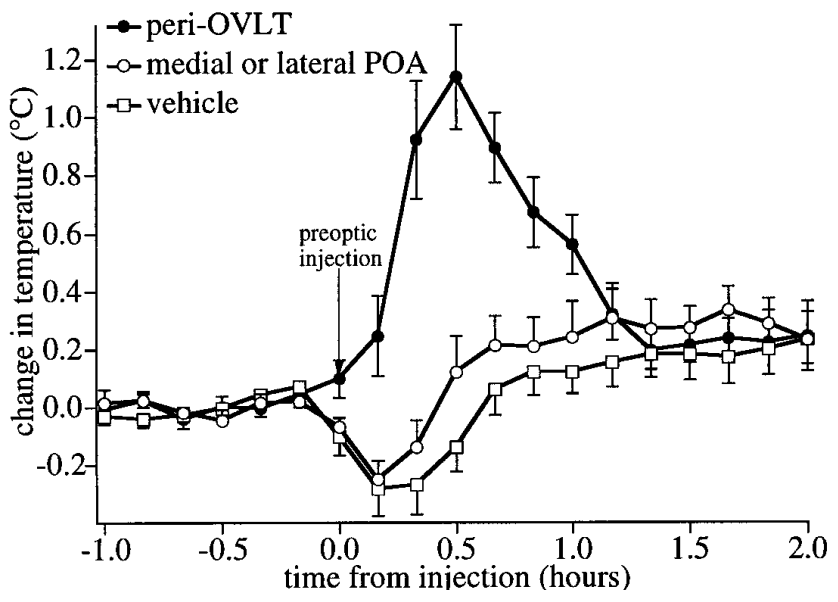

Figure 3. Injection of $1 \mathrm{ng}$ of PGE2 into the ventromedial "pyrogenic zone" rapidly produces large fevers, but injections outside this region or injections of vehicle do not produce fever. Two representative preoptic regions are presented: the peri-OVLT, a region within the pyrogenic zone that consists of the OVLT and most immediately adjacent structures $(n=$ $7)$, and the medial and lateral POAs $(n=10)$ that are outside the pyrogenic zone. Data are presented as the mean change in temperature $( \pm \mathrm{SE})$ in each $10 \mathrm{~min}$ interval relative to baseline (average temperature over the hour preceding injection). 

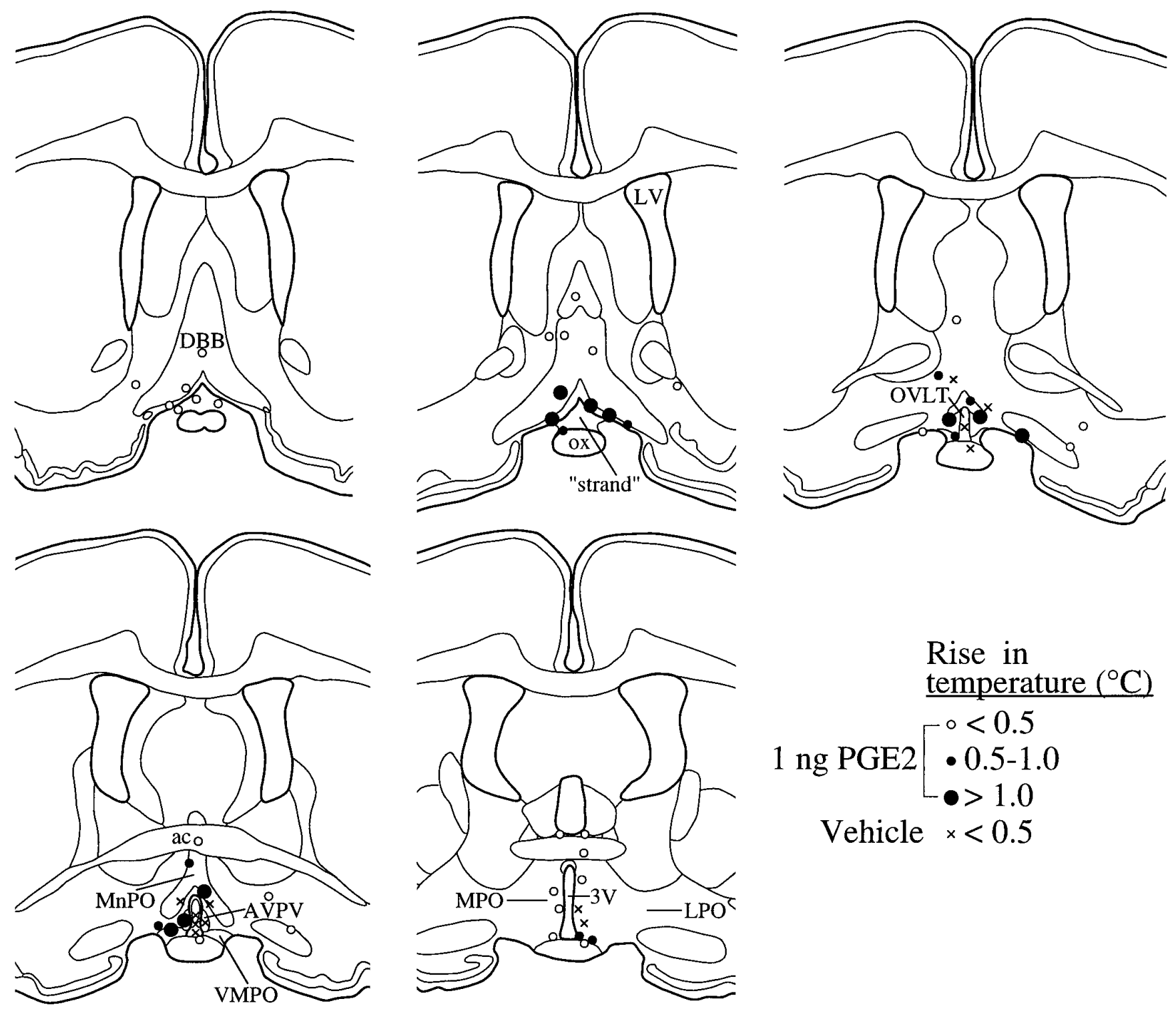

Figure 4. Injection of $1 \mathrm{ng}$ of PGE2 into ventromedial preoptic regions produces fever. Each symbol represents the injection site and corresponding change in Tb 30 min after injection of $1 \mathrm{ng}$ of PGE2 or vehicle. Moderate to large fevers followed PGE2 injections around the OVLT, into the $m n P O$, or between the horizontal nucleus of the diagonal band of Broca $(D B B)$ and the meningeal strand that supports the optic chiasm. ox, Optic chiasm; $L V$, lateral ventricle; $a c$, anterior commissure; $A V P V$, anteroventral periventricular nucleus; $3 V$, third ventricle; $M P O$, medial POA; $L P O$, lateral POA.

cleus consistently produced fevers that often were $>1{ }^{\circ} \mathrm{C}$. PGE2 injections at the edge of this region often produced small fevers, and injections $>300-400 \mu \mathrm{m}$ dorsal or lateral to these regions produced little or no change in $\mathrm{Tb}$. Overall, the most effective PGE2 injections were into the most ventromedial parts of the POA, surrounding the optic recess of the third ventricle. Vehicle injection, even into this "pyrogenic zone," never produced fever.

\section{Sites of PGE2-induced Fos expression}

In our third experiment, we determined the brain regions activated by preoptic injection of a high (100 ng) or low (1 ng) dose of PGE2. The $100 \mathrm{ng}$ injections were scattered throughout the POA and produced large fevers $\left(2.3-3.3^{\circ} \mathrm{C}\right.$ above baseline) that lasted $1.5 \mathrm{hr}$, with ventromedial preoptic injections tending to produce the largest fevers. The $1 \mathrm{ng}$ injections were selected from among the animals described in the previous experiment. Injec- tion of control volumes of vehicle (100 or $10 \mathrm{nl}$ ) had no significant effect on $\mathrm{Tb}$.

\section{General Fos observations}

Fos-IR was seen as a blue-black reaction product that localized to neuronal nuclei. No change in the size or shape of nuclei was evident among groups. Preadsorption of the primary antiserum with Fos peptide or omission of the primary antiserum resulted in no specific staining.

Brains of animals that received $100 \mathrm{nl}$ of vehicle contained a pattern of Fos-IR in many brain regions. Prominent among these was the cerebral cortex (especially the piriform area) ipsilateral to the burr hole. Moderate numbers of immunoreactive cells were seen in the ipsilateral caudate and putamen and in the lateral and central amygdaloid nuclei. A moderate number of immunoreactive cells also were seen bilaterally in and around the suprachias- 


\section{$100 \mathrm{nl}$ vehicle}
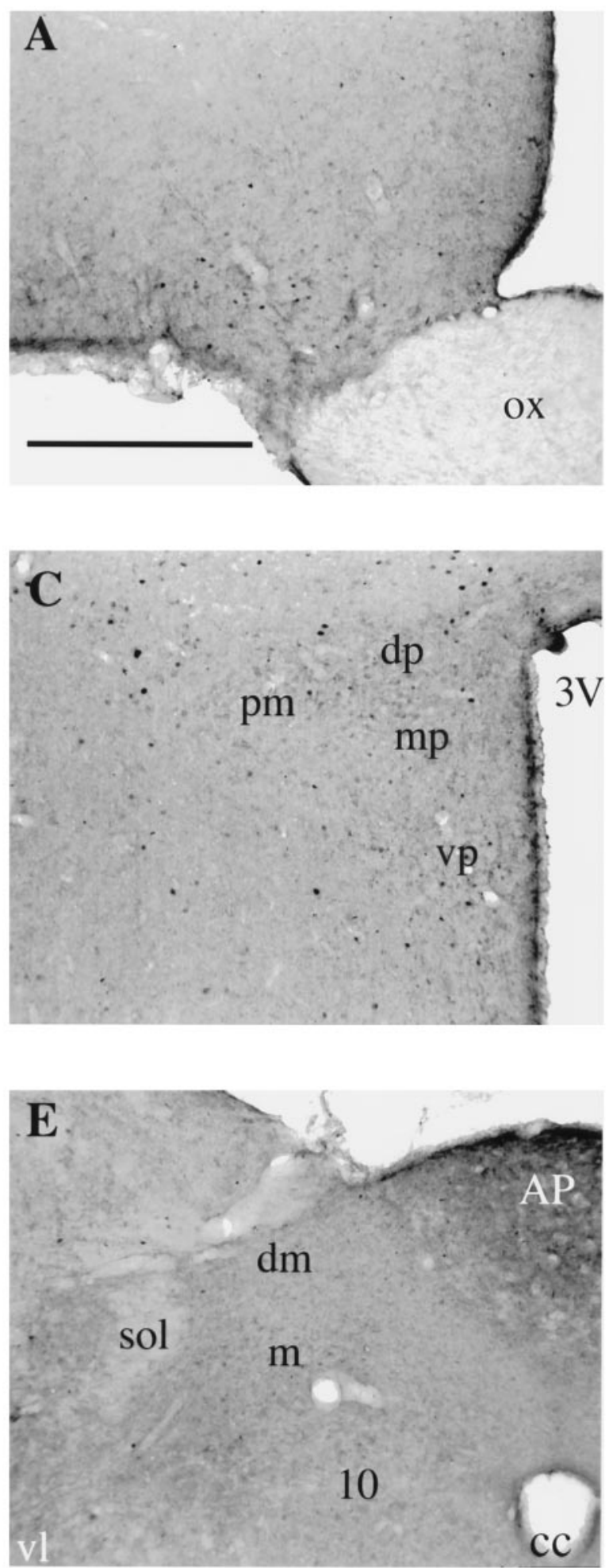

\section{0 ng PGE2}
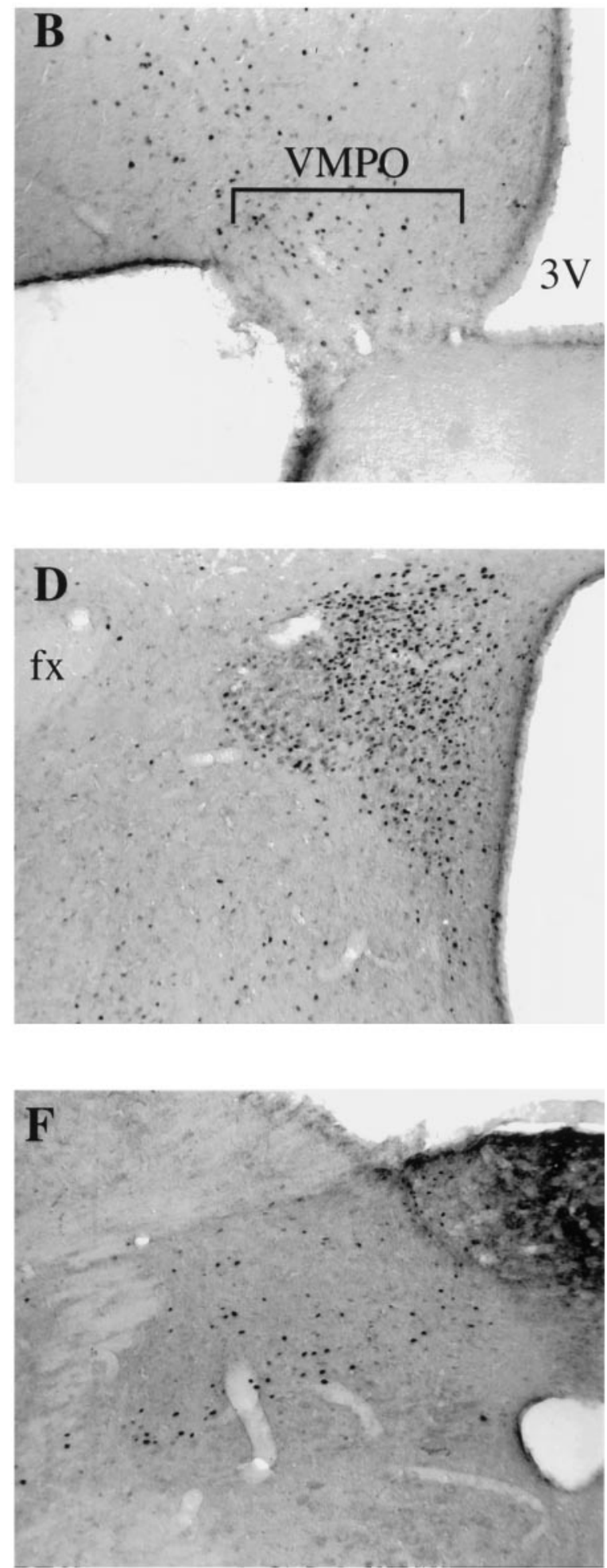

Figure 5. Injections of $100 \mathrm{ng}$ of PGE2 induces Fos-IR in key thermoregulatory regions of rat brain. Bright-field photomicrographs of the VMPO ( $V M P O$, roughly bounded by the bracket) demonstrate little Fos-IR $2 \mathrm{hr}$ after preoptic injection of vehicle $(A)$, but Fos-immunoreactive nuclei are numerous after PGE2 injection $(B)$. Little Fos-IR is seen in the paraventricular nucleus of the hypothalamus after vehicle injection $(C)$, but PGE2 injection strongly induces Fos-IR within the parvicellular, but not the magnocellular, subnuclei $(D)$. After vehicle injection, almost no Fos-IR is visible within the nucleus of the solitary tract $(E)$, but after PGE2 injection, the dorsomedial $(\mathrm{dm})$ and medial $(\mathrm{m})$ subnuclei contain moderate numbers of Fos-immunoreactive nuclei. $p m$, Posterior magnocellular subnucleus; $d p$, dorsal parvicellular subnucleus; $m p$, medial parvicellular subnucleus; $v p$, ventral parvicellular subnucleus; $f x$, fornix; sol, solitary tract; 10 , dorsal motor nucleus of the vagus; $A P$, area postrema; $c c$, central canal. Scale bar, $500 \mu \mathrm{m}$. 
matic nucleus and in the anterior hypothalamic nucleus, the inferior colliculus, the periaqueductal gray matter, the dorsal cochlear nucleus, and the principal sensory and spinal trigeminal nuclei. Moderate numbers of cells were found in the superior lateral, dorsal lateral, and central lateral parabrachial subnuclei and in the ventrolateral medulla (especially the A1/C1 and A1 regions). Immunoreactive cells were rarely observed in the VMPO. Moderate numbers of immunoreactive cells were inconsistently found in the $\mathrm{PVH}$, especially in the medial parvicellular subdivison. Although no histological injury was evident, a thin, $100-\mu \mathrm{m}$-wide column of Fos-immunoreactive neurons extended along the pipette tract in vehicle- and PGE2-injected brains.

\section{PGE2 (100 ng)}

Microinjections of $100 \mathrm{ng}$ of PGE2 into ventromedial preoptic sites produced a striking pattern of Fos-IR superimposed on the pattern attributable to the surgical procedures (Fig. 5). Large numbers of immunoreactive cells were observed bilaterally in the VMPO extending from the level of the OVLT caudally along the ventrolateral aspect of the third ventricle. In one animal that received PGE2 into the cell-sparse zone just below the diagonal band, Fos-IR was found in non-neuronal cells throughout the meninges just below the injection site. A prominent distribution of Fos-IR also was found within the PVH, particularly in the medial parvicellular subdivision, which contains many CRH-producing neurons, and within the dorsal, ventral, and lateral parvicellular subdivisions, which innervate central autonomic structures (Swanson and Sawchenko, 1983; Saper, 1995). The posterior magnocellular subdivision and the supraoptic nuclei contained few immunoreactive cells, except in one animal in which the PGE2 injection was centered on the VMPO, and moderate levels of Fos-IR were seen in the supraoptic nucleus. In the pons, many immunoreactive cells were seen in the lateral parabrachial nucleus, especially in the dorsal and central lateral subnuclei in a pattern similar to controls. In the nucleus of the solitary tract, many cells contained Fos-IR in the medial, dorsomedial, and ventrolateral subnuclei. The lateral edges of the area postrema also contained immunoreactive cells. Fos-IR was bilaterally symmetric in all these affected regions.

To verify these observations more rigorously, we counted the relative number of Fos-immunoreactive neurons in the VMPO, $\mathrm{PVH}$, and NTS of rats that received $100 \mathrm{ng}$ of PGE2 or $100 \mathrm{nl}$ of vehicle (Fig. 6). Rats that received PGE2 had significantly greater numbers of Fos-immunoreactive neurons in the VMPO $(t=-3.8$, $p=0.009)$ and $\mathrm{PVH}(t=-6.03, p=0.001)$ compared with rats that received vehicle. Four times as many Fos-immunoreactive neurons were seen in the NTS of PGE2-treated rats as compared with vehicle-injected rats, but the groups did not differ statistically $(t=-2.87, p=0.053)$.

\section{PGE2 (1 ng)}

We then sought to determine the minimal set of brain regions activated during fever by coupling the threshold dose of PGE2 with Fos histochemistry. Injection of $10 \mathrm{nl}$ of vehicle induced Fos-IR in a pattern indistinguishable from that described above for $100 \mathrm{nl}$ of vehicle (three of these injections were within the pyrogenic zone, four were scattered elsewhere in the POA). Three microinjections of $1 \mathrm{ng}$ of PGE2 outside the pyrogenic zone did not produce fever or induce a distribution of Fos-IR that was notably different from vehicle injections. However, four injections of PGE2 within the pyrogenic zone produced fever and Fos-IR bilaterally in the VMPO; immunoreactive neurons were observed

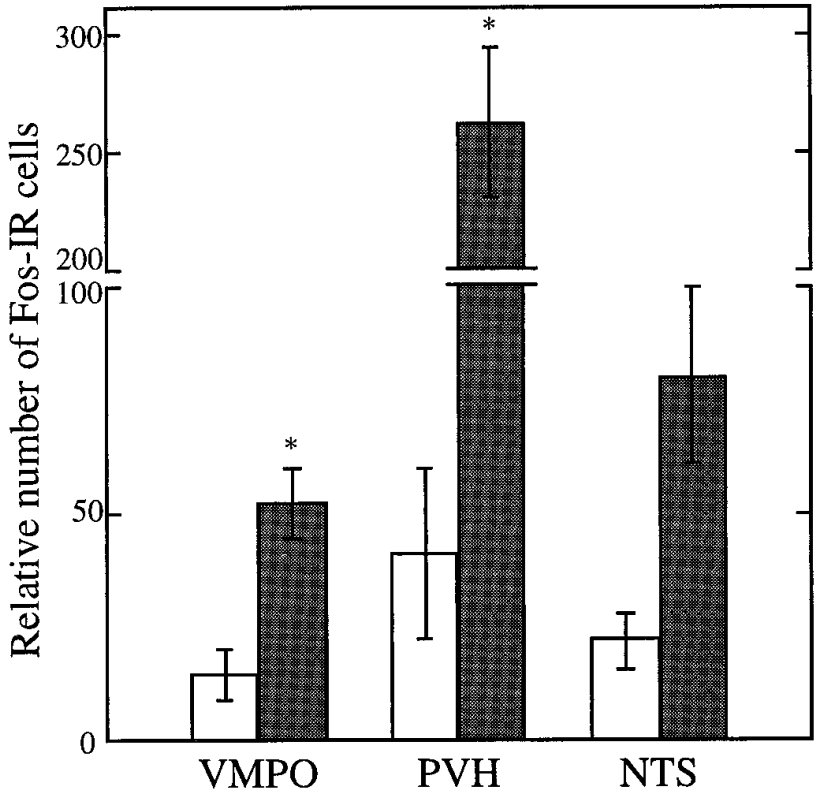

Figure 6. Injection of $100 \mathrm{ng}$ of PGE2 increases the relative number of Fos-immunoreactive nuclei within the $V M P O$ area, $P V H$, and NTS. Open bars represent vehicle-treated rats $(n=3)$, and filled bars represent rats treated with $100 \mathrm{ng}$ of PGE2 $(n=5) ;{ }^{*} p<0.01$.

consistently throughout the rostro-caudal extent of the VMPO, although the number of cells was considerably fewer than the number seen after $100 \mathrm{ng}$ of PGE2 (Fig. 7). Additionally, increased but variable numbers of Fos-immunoreactive neurons were seen in the dorsal and ventral parvicellular subnuclei of the PVH. No consistent Fos-IR was seen in the NTS or any other autonomic nuclear groups.

Because the VMPO is close to many of the effective feverproducing injection sites, we performed a two-way ANOVA to determine the relative influences of PGE2 and injection sites. Neither drug treatment (PGE2 vs vehicle) nor injection site (inside vs outside the pyrogenic zone) in itself correlated with the induction of Fos-IR in the VMPO. Only PGE2 injections into the pyrogenic zone significantly increased the number of Fos-immunoreactive neurons in the VMPO $(p=0.03)$ (Fig. 8).

\section{DISCUSSION}

We found that microinjections of a threshold dose of PGE2 into the POA rapidly induces fever, and the most pyrogenic preoptic sites are clustered along the ventromedial aspect of the POA, surrounding and anterior to the OVLT. This PGE2 fever is accompanied by Fos induction in the VMPO and the autonomic regulatory and $\mathrm{CRH}$-producing subdivisions of the $\mathrm{PVH}$. These results suggest that during the acute-phase reaction, PGE2 may activate the VMPO, which, in turn, stimulates the PVH to produce fever.

\section{Methodological considerations}

Our injection technique has allowed us to construct a detailed map of pyrogenic sites in the POA. Compared with previous studies, we used very low doses and volumes of PGE2 to stimulate the smallest possible regions and obtain maximal anatomical precision. Previous investigations all used chronic injection cannulae or studied PGE2 fever in anesthetized animals. During sustained general anesthesia, animals often require considerably more PGE2 to develop fever, possibly because of anesthesia- 


\section{$10 \mathrm{nl}$ vehicle}
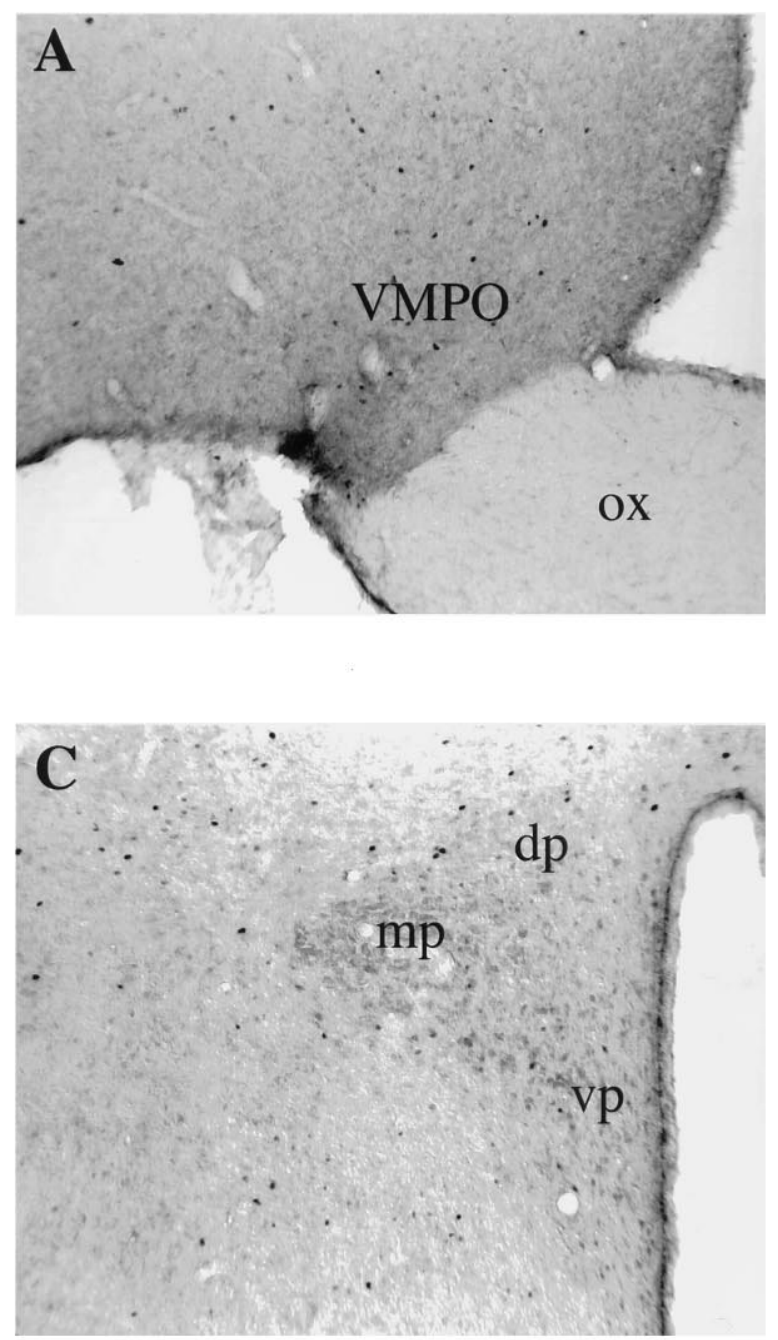

\section{1 ng PGE2}
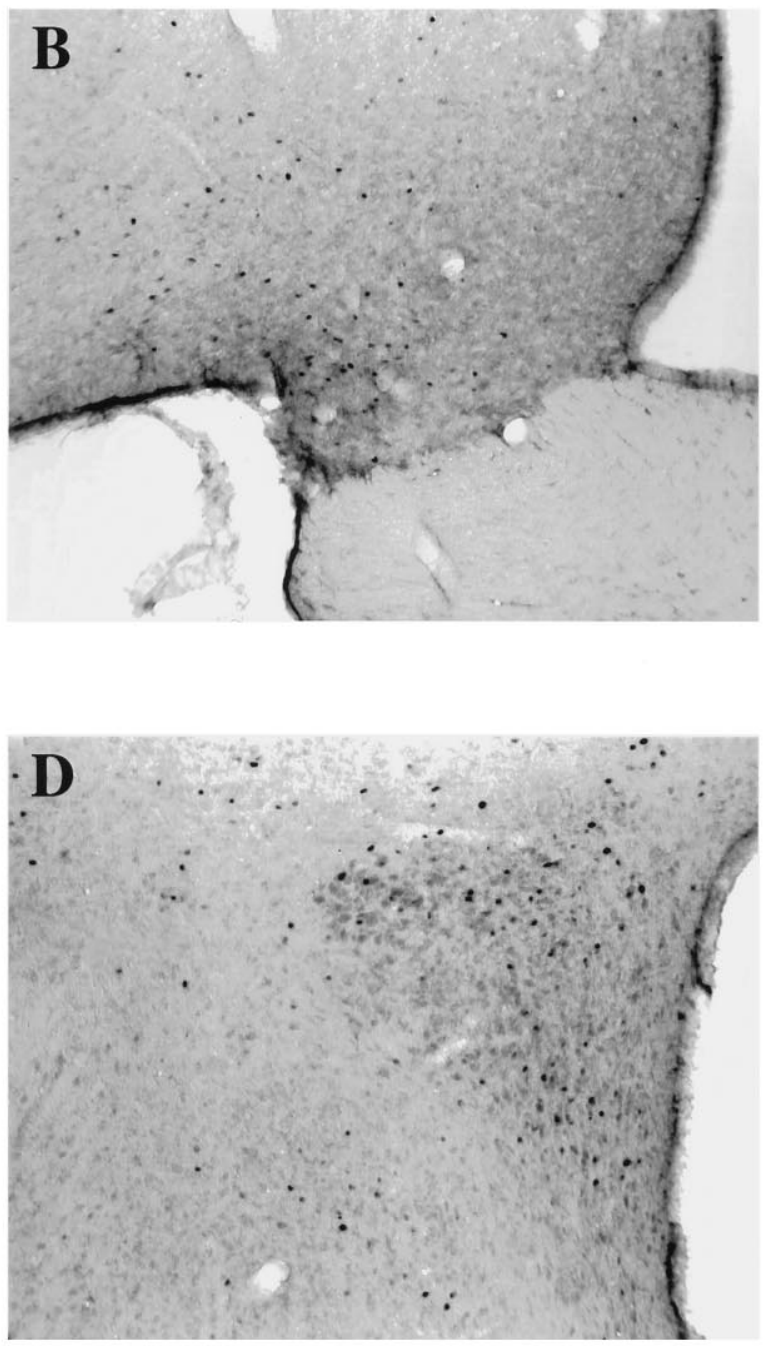

Figure 7. Injection of $1 \mathrm{ng}$ of PGE2 induces Fos-IR in the VMPO and PVH. Rare Fos-immunoreactive nuclei are evident in the VMPO after vehicle injection $(A)$, but a moderate number of nuclei are seen after PGE2 injection $(B)$. The PVH typically has few Fos-immunoreactive nuclei after vehicle injection $(C)$, but occasional Fos-IR is seen in the dorsal $(d p)$, medial $(m p)$, and ventral parvicellular $(v p)$ subnuclei after PGE2 injection $(D)$.

induced thermoregulatory dysfunction (Feldberg and Saxena, 1971). In contrast, the 10-15 min period of anesthesia in our preparation had little effect on thermoregulation. Furthermore, chronic cannulae may induce local PGE2 production (Yergey and Heyes, 1990) such that large amounts of PGE2 are subsequently required for physiological effects. These technical concerns may be why most previous studies of PGE2-induced fever required 50-100 ng of PGE2 to produce reliable fevers. Additionally, high doses of PGE2 may have been required in some studies, because the injections were too far from the pyrogenic ventromedial preoptic region. Stitt found that $1 \mathrm{ng}$ of PGE2 injected close to the OVLT reliably produced $0.8^{\circ} \mathrm{C}$ fevers (Stitt, 1991), but the large volume injected $(1 \mu \mathrm{l})$ and the trauma produced by the chronic cannulae preclude a detailed anatomic interpretation. Our technique of acute injections under brief anesthesia followed by careful injection-site identification allowed us to use small volumes of PGE2 to obtain high anatomic resolution; highly effective feverproducing sites were separated from ineffective sites by only 200-300 $\mu \mathrm{m}$.
The coupling of brain injections with Fos immunohistochemistry allows us to identify candidate circuits involved in the production of fever but also raises several concerns. First, the stereotaxic surgical technique itself induces Fos-IR in a number of brain regions. We and others have noted that the injection procedure itself induces Fos-IR throughout much of the ipsilateral cortex, sensory trigeminal nuclei, and cochlear nuclei (Sharp et al., 1990; Krukoff et al., 1992; Amir et al., 1994). Fos induction in the cerebral cortex may be caused by glutamate-mediated spreading depression (Sharp et al., 1990), whereas Fos in sensory nuclei is more likely caused by meningeal and middle ear trauma secondary to the stereotaxic surgical technique itself. We commonly observed a thin column of Fos-immunoreactive neurons along the pipette tract that hinders interpretation of the Fos pattern in the immediate vicinity of the injection site, but as shown in the final experiment, functionally meaningful Fos expression can be studied in structures a few hundred microns away. Second, general anesthesia can induce Fos in autonomic regulatory regions; several authors have described Fos-IR in the medial POA, PVH 


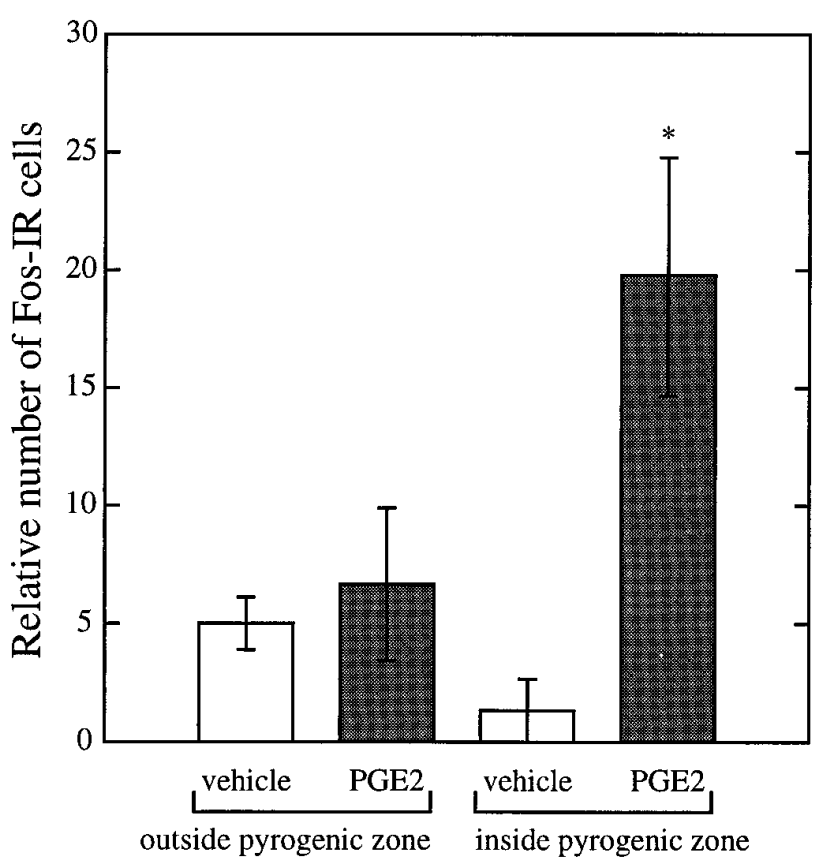

Figure 8. Injection of $1 \mathrm{ng}$ of PGE2 into the pyrogenic zone induces Fos-IR in the VMPO. Injection of PGE2 outside the pyrogenic zone or injection of vehicle even into the pyrogenic zone fails to induce much Fos-IR ( $n=3-4$ in each group; $\left.{ }^{*} p=0.03\right)$.

(primarily the parvicellular regions), ventromedial hypothalamus, lateral parabrachial nucleus, NTS, and ventrolateral medulla (VLM) after anesthesia with long-acting agents (Millhorn, 1991; Krukoff et al., 1992; Erickson and Dampney et al., 1995). We also found that vehicle injection under brief propofol anesthesia induced moderate Fos-IR in the parabrachial nucleus and VLM, but Fos-immunoreactive neurons were uncommon in the NTS, $\mathrm{PVH}$, and POA. We suspect that propofol anesthesia is too brief and the autonomic changes too mild to induce Fos in these latter regions. After anesthesia with propofol, Fos-IR needs to be viewed critically, but in areas little affected by anesthesia and the injection technique, Fos is a useful indicator of which neuronal groups may contribute to the production of fever.

Conversely, the absence of Fos-IR cannot be used to exclude functional participation of a nuclear group. Even excitatory responses leading to neuronal activation may have different thresholds for inducing Fos in different brain regions (Ericsson et al., 1994). We had hypothesized that a $1 \mathrm{ng}$ threshold dose of PGE2 would induce Fos in several brain regions essential for fever, but a notable effect was evident only in the VMPO and, to a lesser degree, in autonomic regulatory regions of the $\mathrm{PVH}$. Although the VMPO may play a central role in fever, we suspect that additional autonomic control regions must contribute to the production of fever; a threshold dose of PGE2 may be too weak a stimulus to reliably induce Fos in distantly activated regions.

\section{The "pyrogenic zone"}

PGE2 injections into the most ventromedial regions of the POA were most effective at producing fever. This ventromedial pyrogenic zone surrounds the OVLT, a highly vascular structure that has been hypothesized to be the site at which circulating cytokines induce PGE2 production to initiate fever (Stitt, 1991). The prostaglandin-synthesizing enzyme cyclooxygenase- 2 is induced in microglial cells along blood vessels in the OVLT and adjacent meninges after systemic (intravenous) immune stimulation with the bacterial cell wall component lipopolysaccharide (LPS) (C. Breder and C. Saper, unpublished observations). The adjacent pyrogenic zone correlates well with the high concentration of PGE2 binding sites that surround the OVLT and extend dorsally into the mnPO nucleus and laterally into the VMPO (Matsumura et al., 1990). Using in situ hybridization, Ericsson recently described a similar pattern in the distribution of the EP3 type of PGE2 receptor that may be important in fever (Ericsson et al., 1995). Although PGE2 may be synthesized within the OVLT, our observations, combined with the PGE2 binding studies and early receptor localization work, suggest that PGE2 produces fever by acting in preoptic regions surrounding the OVLT.

\section{Fos pattern}

Many autonomic regulatory structures are activated during PGE2 fever in a pattern similar to that seen during the fever produced by systemic administration of LPS. Both intravenous LPS (Elmquist et al., 1996) and $100 \mathrm{ng}$ of intrapreoptic PGE2 induce Fos in the VMPO, mnPO nucleus, parvicellular areas of the PVH, and NTS. The most robust response among these regions is within the VMPO where Fos is induced at threshold pyrogenic doses of either LPS or PGE2.

The Fos pattern induced by intrapreoptic PGE2 differs from that seen with LPS in several notable respects. In contrast to LPS, PGE2 does not induce Fos within magnocellular PVH neurons or within the core regions of three circumventricular organs (OVLT, subfornical organ, and area postrema). These differences most likely are attributable to the different route of action and effects of LPS. First, intravenous LPS may act at circumventricular organs to produce many of the brain-mediated aspects of the acute-phase response (Saper and Breder, 1994; Elmquist et al., 1996). Second, high doses of LPS induce hypotension and subsequent release of vasopressin and oxytocin (Aiura et al., 1995); most likely, the marked induction of Fos in magnocellular neurons occurs during the increased firing that causes peptide secretion.

We hypothesize that the VMPO may be an essential link in the production of fever; PGE2 (either produced near the OVLT after LPS or directly injected) may stimulate neurons in the VMPO that alter the thermoregulatory setpoint by means of projections to the $\mathrm{PVH}$ and other autonomic regulatory regions. We have stressed the potential role of the VMPO, because unlike other $\mathrm{PVH}$-projecting regions of the pyrogenic zone such as the anteroventral periventricular nucleus and the mnPO nucleus (Saper and Levisohn, 1983; Simerly and Swanson, 1988; Standaert and Saper, 1988), the VMPO reliably produces Fos during LPS- and PGE2induced fever. VMPO neurons directly project to the dorsal and ventral parvocellular PVH subnuclei, areas that regulate autonomic function, and these PVH-projecting VMPO neurons are activated during fever (Elmquist and Saper, 1996). In addition, we are studying other inputs from the VMPO to the PVH that may be relayed via the anterior perifornical region (Elmquist et al., 1995).

The PVH is well positioned to coordinate the neuroendocrine and autonomic activity required for the production of fever. The $\mathrm{PVH}$ projects directly to preganglionic sympathetic and parasympathetic neurons as well as to sympathetic premotor sites in the parabrachial nucleus, VLM, and NTS (Swanson and Sawchenko, 1983; Saper, 1995). Thus, through its connections with the PVH, the VMPO may contribute to the increased sympathetic activity and redistributed blood flow required for the production of fever (for detailed discussion, see Elmquist et al., 1996). 


\section{Perspective}

We have demonstrated that threshold doses of PGE2 produce fever when injected into the ventromedial pyrogenic zone of the POA. These PGE2 fevers are accompanied by activation of neurons in the VMPO and other key autonomic regulatory areas in a pattern similar to that seen during the fever produced by intravenous LPS. These observations establish a potential site of PGE2 action during fever and help define the central pathways through which fever is mediated. However, it remains to be established whether PGE2 is necessary for fever and, if so, through which specific PGE2 receptors. Future experiments to block PGE2 production or selectively antagonize its action will greatly aid in clarifying the neural mechanisms of fever and the broader role of the brain in the acute-phase reaction.

\section{REFERENCES}

Aiura K, Ueda M, Endo M, Kitajima M (1995) Circulating concentrations and physiological role of atrial natriuretic peptide during endotoxic shock in the rat. Crit Care Med 23:1898-1906.

Amaral DG, Price JL (1983) An air pressure system for the injection of tracer substances into the brain. J Neurosci Methods 9:35-43.

Amir S, Robinson B, Woodside B (1994) Induction of Fos protein in the piriform cortex after brain injury in pentobarbital-anaesthetized rats: lack of effect of lactation. Brain Res 652:341-345.

Breder CD, Saper CB (1996) Expression of inducible cyclooxygenase mRNA in the mouse brain after systemic administration of bacterial lipopolysaccharide. Brain Res 713:64-69.

Dampney RA, Li YW, Hirooka Y, Potts P, Polson JW (1995) Use of c-fos functional mapping to identify the central baroreceptor reflex pathway: advantages and limitations. Clin Exp Hypertens [A] 17:197-208.

Elmquist JK, Saper CB (1996) Activation of neurons projecting to the paraventricular nucleus by intravenous lipopolysaccharide. J Comp Neurol, in press.

Elmquist JK, Scammell TE, Jacobson CD, Saper CB (1996) Distribution of Fos-like immunoreactivity in the rat brain following intravenous lipopolysaccharide. J Comp Neurol 371:85-103.

Elmquist JK, Scammell TE, Saper CB (1995) Anatomic characterization of the ventral medial preoptic area: involvement in the febrile reaction. Paper presented at the Annual Meeting of the Society for Neuroscience, San Diego, November.

Erickson JT, Millhorn DE (1991) Fos-like protein is induced in neurons of the medulla oblongata after stimulation of the carotid sinus nerve in awake and anesthetized rats. Brain Res 567:11-24.

Ericsson A, Kovacs KJ, Sawchenko PE (1994) A functional anatomical analysis of central pathways subserving the effects of interleukin-1 on stress-related neuroendocrine neurons. J Neurosci 14:897-913.

Ericsson A, Ek M, Lindefors N (1995) Distribution of prostaglandin E2 receptor (EP3 subtype) mRNA-containing cells in the rat central nervous system. Paper presented at the Annual Meeting of the Society for Neuroscience, San Diego, CA, November.

Feldberg W, Saxena PN (1971) Further studies on prostaglandin E1 fever in cats. J Physiol (Lond) 219:739-745.
Kluger MJ (1991) Fever: role of pyrogens and cryogens. Physiol Rev 71:93-127.

Konigsmark BW (1970) Methods for the counting of neurons. In: Contemporary research methods in neuroanatomy (Nauta WJH, Ebbesson SOE, eds), pp 315-338. Heidelberg: Springer.

Krukoff TL, Morton TL, Harris KH, Jhamandas JH (1992) Expression of c-fos protein in rat brain elicited by electrical stimulation of the pontine parabrachial nucleus. J Neurosci 12:3582-3590.

Larsson JE, Wahlstrom G (1994) Optimum rate of administration of propofol for induction of anaesthesia in rats. Br J Anaesth 73:692-694.

Matsumura K, Watanabe Y, Onoe H, Watanabe Y, Hayaishi O (1990) High density of prostaglandin E2 binding sites in the anterior wall of the 3rd ventricle: a possible site of its hyperthermic action. Brain Res 533:147-151

Morgan JI, Curran T (1991) Stimulus-transcription coupling in the nervous system: involvement of the inducible proto-oncogenes fos and jun. Annu Rev Neurosci 14:421-451.

Paxinos G, Watson C (1986) The rat brain in stereotaxic coordinates. New York: Academic.

Sagar SM (1994) The functional neuroanatomy of the acute-phase response. Ann NY Acad Sci 739:282-291.

Sagar SM, Sharp FR, Curran T (1988) Expression of c-fos protein in brain: metabolic mapping at the cellular level. Science 240:1328-1331.

Saper CB (1995) Central autonomic system. In: The rat nervous system (Paxinos G, ed), pp 107-135. New York: Academic.

Saper CB, Breder CD (1994) The neurologic basis of fever. N Engl J Med 330:1880-1886.

Saper CB, Levisohn D (1983) Afferent connection of the median preoptic nucleus in the rat: anatomical evidence for a cardiovascular integrative mechanism in the anteroventral third ventricular (AV3V) region. Brain Res 288:21-31.

Sharp JW, Sagar SM, Hisanaga K, Jasper P, Sharp FR (1990) The NMDA receptor mediates cortical induction of fos and fos-related antigens following cortical injury. Exp Neurol 109:323-332.

Simerly RB, Swanson LW (1988) Projections of the medial preoptic nucleus: a Phaseolus vulgaris leucoagglutinin anterograde tract-tracing study in the rat. J Comp Neurol 270:209-242.

Standaert DG, Saper CB (1988) Origin of the atriopeptin-like immunoreactive innervation of the paraventricular nucleus of the hypothalamus. J Neurosci 8:1940-1950.

Stitt J (1991) Differential sensitivity in the sites of fever production by prostaglandin E2 within the hypothalamus of the rat. J Physiol (Lond) 432:99-110.

Swanson LW, Sawchenko PE (1983) Hypothalamic integration: organization of the paraventricular and supraoptic nuclei. Annu Rev Neurosci 6:269-324.

Vane JR (1971) Inhibition of prostaglandin synthesis as a mechanism of action for aspirin-like drugs. Nat New Biol 231:232-235.

Williams JW, Rudy TA, Yaksh TL, Viswanathan CT (1977) An extensive exploration of the rat brain for sites mediating prostaglandin-induced hyperthermia. Brain Res 120:251-262.

Yergey JA, Heyes MP (1990) Brain eicosanoid formation following acute penetration injury as studied by in vivo microdialysis. J Cereb Blood Flow Metab 10:143-146. 